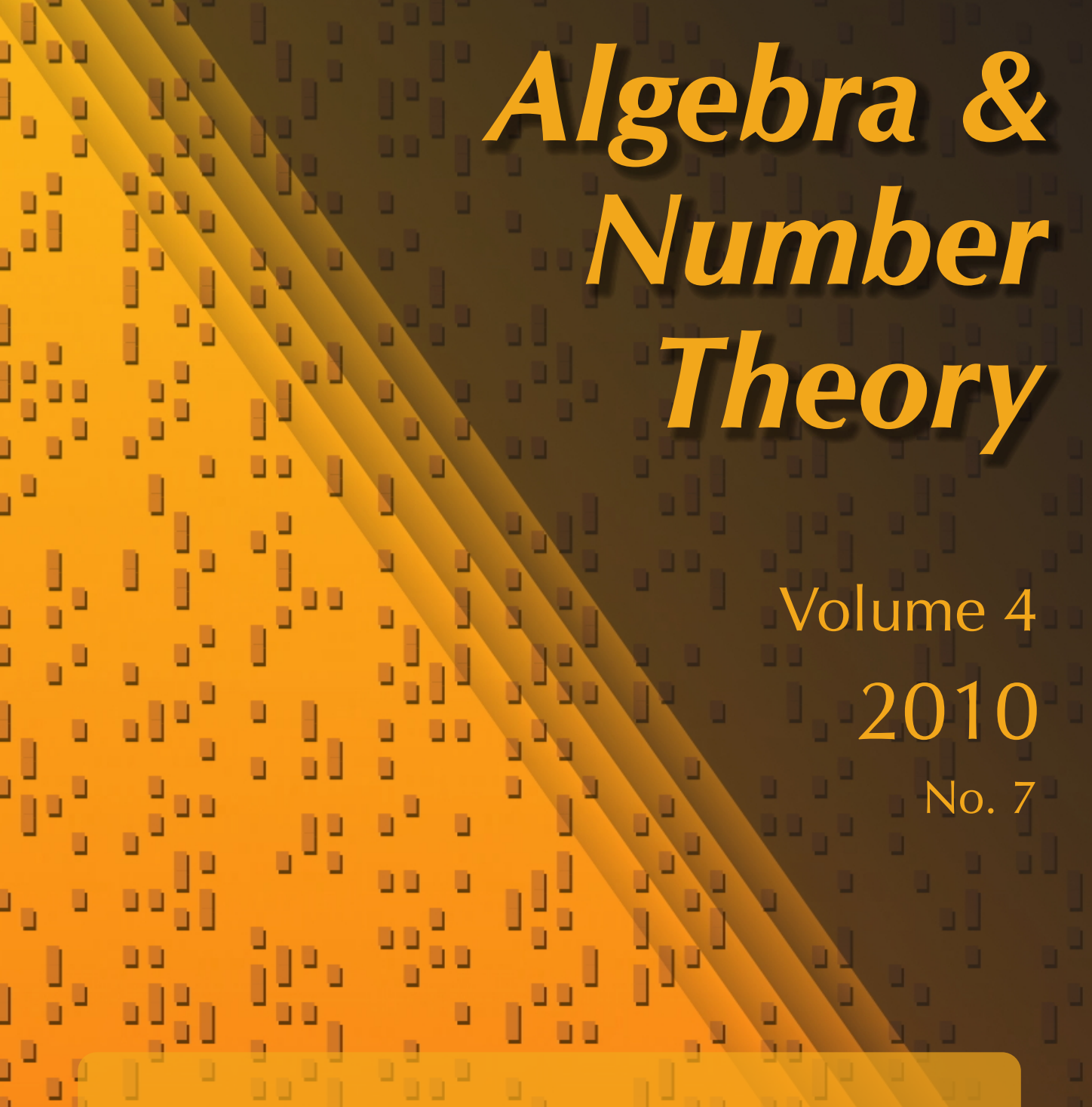

Hochschild cohomology and homology of quantum

\lrcorner$\lrcorner \quad$ Steffen Oppermann complete intersections $\perp+$

」 


\title{
Hochschild cohomology and homology of quantum complete intersections
}

\author{
Steffen Oppermann
}

\begin{abstract}
We compute the Hochschild cohomology and homology for arbitrary finitedimensional quantum complete intersections. It turns out that their behavior varies widely, depending on the choice of commutation parameters, and we give precise criteria for when to expect what behavior.
\end{abstract}

\section{Introduction}

Quantum complete intersections were first discussed by Avramov, Gasharov, and Peeva [Avramov et al. 1997]. Based on the introduction of quantized versions of polynomial rings in [Manin 1987], they introduced the notion of quantum regular sequences.

In this paper we restrict to finite-dimensional quantum complete intersections, that is, algebras of the form $k\left\langle x_{1}, \ldots, x_{c}\right\rangle / I$, where $I$ is an ideal generated by $x_{i}^{n_{i}}$ for some $n_{i} \in \mathbb{N}_{\geq 2}$, and $x_{j} x_{i}-q_{i j} x_{i} x_{j}$ for some commutation parameters $q_{i j}$ from the multiplicative group of the field.

In particular, in the case of two variables it is known that the homological behavior of finite-dimensional quantum complete intersections varies greatly, depending on the commutation parameters.

Buchweitz, Green, Madsen, and Solberg [Buchweitz et al. 2005] gave a finitedimensional quantum complete intersection as the first example of an algebra of infinite global dimension which has finite Hochschild cohomology. This result was generalized in [Bergh and Erdmann 2008], which showed that a finite-dimensional quantum complete intersection of codimension 2 ( $c=2$ in the description above) has an infinite Hochschild cohomology if and only if the commutation parameter is a root of unity.

On the other hand, in [Bergh and Oppermann 2008a] we showed that in the situation that all commutation parameters are roots of unity, the Hochschild cohomology of a quantum complete intersection is as well behaved as in the commutative case:

The author was supported by NFR Storforsk grant no. 167130 .

MSC2000: primary 16E40; secondary 81R50, 16U80, 16S80.

Keywords: Hochschild cohomology, Hochschild homology, quantum complete intersection. 
It is a finitely generated $k$ algebra, and any $\operatorname{Ext}^{*}(M, N)$ for any finite-dimensional modules $M$ and $N$ over the quantum complete intersection is finitely generated as a module over the Hochschild cohomology ring.

This paper gives a general description of the Hochschild cohomology and homology of finite-dimensional quantum complete intersections. Here is an outline:

In Theorems 3.4 and 7.4 we explicitly determine a $k$-basis for the Hochschild cohomology and homology, respectively.

Using these results we study the size of the Hochschild cohomology and homology in the following sense: Let $\mathbb{N}$ be the set of nonnegative integers (i.e., $0 \in \mathbb{N}$ ). We denote by

$$
\gamma\left(\mathrm{HH}^{*}(\Lambda)\right)=\inf \left\{t \in \mathbb{N} \mid \limsup \frac{\operatorname{dim}_{k} \operatorname{HH}^{n}(\Lambda)}{n^{t-1}}<\infty\right\}
$$

the rate of growth of the Hochschild cohomology (and similarly for the Hochschild homology). In Theorems 4.5 and 8.2, we obtain explicit combinatorial formulas for $\gamma\left(\mathrm{HH}^{*}(\Lambda)\right)$ and $\gamma\left(\mathrm{HH}_{*}(\Lambda)\right)$. In particular it will be shown (Corollary 4.6) that whenever not all commutation parameters are roots of unity we have $\gamma\left(\operatorname{HH}^{*}(\Lambda)\right) \leq$ $c-2$. For $c=2$ that means that the Hochschild cohomology is finite. This explains why there are essentially only two cases for $c=2$, while we obtain additional behaviors for larger $c$.

We will also generalize the result of [Bergh and Erdmann 2008] in another way: It will be shown that whenever the commutation parameters are sufficiently generic the Hochschild cohomology of the quantum complete intersection is finite (see Example 6.2).

Finally we will study the multiplicative structure of the Hochschild cohomology ring. It will turn out (Theorem 5.5) that it always contains a subring $\mathscr{S}$ which is finitely generated over $k$, and isomorphic to the quotient of the Hochschild cohomology modulo its nilpotent elements. We will give a criterion for when the entire Hochschild cohomology ring is finitely generated over this subring (Theorem 5.9). We will give examples (Examples 6.4 and 6.5) that all the following behaviors occur (for $c \geq 3$ ):

- $\mathscr{Y}=k$, but $\gamma\left(\mathrm{HH}^{*}(\Lambda)\right)=c-2$.

- $\gamma(\mathscr{S})=\gamma\left(\mathrm{HH}^{*}(\Lambda)\right)=c-2$, and $\mathrm{HH}^{*}(\Lambda)$ is finitely generated over $\mathscr{Y}$.

- $\gamma(\mathscr{S})=\gamma\left(\mathrm{HH}^{*}(\Lambda)\right)=c-2$, but $\mathrm{HH}^{*}(\Lambda)$ is not finitely generated over $\mathscr{S}$.

\section{Notation and background}

Throughout this paper we assume $k$ to be field.

Quantum complete intersections. (See also [Bergh and Erdmann 2008; Bergh and Oppermann 2008a; 2008b].) A finite-dimensional quantum complete intersection 
of codimension $c$ is a $k$-algebra of the form

$$
\Lambda_{\boldsymbol{q}}^{\boldsymbol{n}}=\frac{k\left\langle x_{1}, \ldots, x_{c}\right\rangle}{\left(\begin{array}{cl}
x_{i}^{n_{i}} & \text { for } 1 \leq i \leq c \\
x_{j} x_{i}-q_{i j} x_{i} x_{j} & \text { for } 1 \leq i<j \leq c
\end{array}\right)}
$$

with $\boldsymbol{n}=\left(n_{1}, \ldots, n_{c}\right) \in \mathbb{N}_{\geq 2}^{c}$ and $\boldsymbol{q}=\left(q_{i j} \mid i<j\right) \in\left(k^{\times}\right)^{n(n-1) / 2}$, where $k^{\times}$denotes the multiplicative group $k \backslash\{0\}$. For convenience of notation we also define $q_{i j}$ for $i \geq j$ : We set $q_{i i}=1$ for any $i \in\{1, \ldots, c\}$ and $q_{i j}=q_{j i}^{-1}$ for $1 \leq j<i \leq c$. The relations $x_{j} x_{i}-q_{i j} x_{i} x_{j}$ for $1 \leq j \leq i \leq c$ are automatically satisfied in $\Lambda_{q}^{n}$.

Note that $\Lambda_{q}^{n}$ is a $\mathbb{Z}^{c}$-graded algebra by $\left|x_{i}\right|=$ degree $x_{i}=e_{i}$, the $i$-th unit vector. We will denote by $\leq$ the partial order on $\mathbb{Z}^{c}$ defined by comparing vectors componentwise, and by $\mathbf{1}=\sum e_{i}$ the vector with 1 in every component. With this notation, the dimensions of the graded component of degree $\boldsymbol{d}$ (with $\boldsymbol{d} \in \mathbb{Z}^{c}$ ) are

$$
\operatorname{dim}\left(\Lambda_{q}^{\boldsymbol{n}}\right)_{\boldsymbol{d}}= \begin{cases}1 & \text { if } \mathbf{0} \leq \boldsymbol{d} \leq \boldsymbol{n}-\mathbf{1} \\ 0 & \text { otherwise }\end{cases}
$$

For $\boldsymbol{a} \in \mathbb{N}^{c}$ we will write $\boldsymbol{x}^{\boldsymbol{a}}=x_{1}^{a_{1}} \ldots x_{c}^{a_{c}}$. Note that the multiplication yields something different if we multiply in another order. In particular we do not have $x^{a} x^{b}=x^{a+b}$. By setting

$$
\boldsymbol{q}^{\langle\boldsymbol{a} \mid \boldsymbol{b}\rangle}=\prod_{\substack{i, j \in\{1 \ldots c\} \\ i<j}} q_{i j}^{a_{j} b_{i}}
$$

we obtain the multiplication formula $x^{a} x^{b}=q^{\langle a \mid b\rangle} x^{a+b}$.

Hochschild (co)homology. Let $\Lambda$ be a finite-dimensional algebra. We denote by $\Lambda^{\mathrm{en}}=\Lambda \otimes_{k} \Lambda^{\text {op }}$ the enveloping algebra. Then $\Lambda^{\mathrm{en}}$-modules are $\Lambda$ - $\Lambda$ bimodules on which $k$ acts centrally. In particular $\Lambda$ has a natural structure of a $\Lambda^{\text {en }}$-module. Then

$$
\operatorname{HH}^{*}(\Lambda)=\operatorname{Ext}_{\Lambda^{\text {en }}}^{*}(\Lambda, \Lambda) \text { and } \operatorname{HH}_{*}(\Lambda)=\operatorname{Tor}_{*}^{\Lambda^{\text {en }}}(\Lambda, \Lambda)
$$

are the Hochschild cohomology and Hochschild homology of $\Lambda$, respectively. With the Yoneda multiplication of extensions $\mathrm{HH}^{*}$ becomes a $\mathbb{Z}$-graded $k$-algebra, which is graded commutative [Yoneda 1958].

If $\Lambda$ is graded then so is $\Lambda^{\mathrm{en}}$, and $\Lambda$ is a graded $\Lambda^{\mathrm{en}}$-module. It follows that for any $i \in \mathbb{N}$ the Hochschild homology and cohomology groups $\mathrm{HH}_{i}(\Lambda)$ and $\mathrm{HH}^{i}(\Lambda)$ are also graded.

Projective resolutions. In order to determine the Hochschild homology and cohomology of a quantum complete intersection $\Lambda=\Lambda_{q}^{n}$ we need to find a projective resolution of $\Lambda$ as a $\Lambda^{\text {en }}$-module. Moreover we want to keep track of the $\mathbb{Z}^{c}$-grading, so we will need a graded projective resolution. 
We have shown in [Bergh and Oppermann 2008a, Lemma 4.5] that such a graded projective resolution can be found by tensoring together the projective resolutions of $k\left[x_{i}\right] /\left(x_{i}^{n_{i}}\right)$ as $\left(k\left[x_{i}\right] /\left(x_{i}^{n_{i}}\right)\right)^{\text {en }}$ modules. To simplify the notation we set $\Lambda_{i}=k\left[x_{i}\right] /\left(x_{i}^{n_{i}}\right)$. Then the graded projective resolution of $\Lambda_{i}$ as a bimodule is

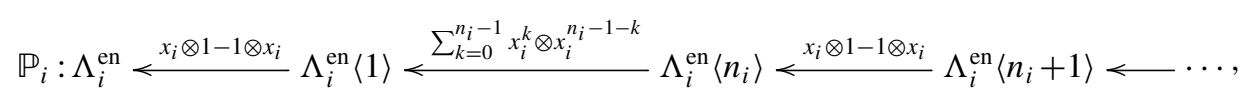

where $\Lambda_{i}^{\text {en }}\langle s\rangle$ is the graded module obtained from $\Lambda_{i}^{\text {en }}$ by increasing the degree of all homogeneous elements by $s$. Note that here all the bimodules are shifted into place in such a way that all the morphisms have degree 0 .

With this notation, the total complex

$$
\operatorname{Tot}\left(\mathbb{P}_{1} \otimes_{k} \mathbb{P}_{2} \otimes_{k} \cdots \otimes_{k} \mathbb{P}_{c}\right)
$$

is a graded projective resolution of $\Lambda$.

The term in position $\boldsymbol{p} \in \mathbb{N}^{c}$ of the $c$-tuple complex $\mathbb{P}_{1} \otimes_{k} \mathbb{P}_{2} \otimes_{k} \cdots \otimes_{k} \mathbb{P}_{c}$ is

$$
\Lambda_{1}^{\mathrm{en}}\left\langle\mathfrak{s}(\boldsymbol{p})_{1}\right\rangle \otimes \cdots \otimes \Lambda_{c}^{\mathrm{en}}\left\langle\mathfrak{s}(\boldsymbol{p})_{c}\right\rangle,
$$

where for notational compactness we have defined a function $\mathfrak{s}: \mathbb{Z}^{c} \rightarrow \mathbb{Z}^{c}$ by

$$
\mathfrak{s}(\boldsymbol{p})_{i}= \begin{cases}\frac{1}{2} p_{i} n_{i} & \text { if } p_{i} \text { is even, } \\ \frac{1}{2}\left(p_{i}-1\right) n_{i}+1 & \text { if } p_{i} \text { is odd. }\end{cases}
$$

We will also need a left inverse $\mathfrak{p}: \mathbb{Z}^{c} \rightarrow \mathbb{Z}^{c}$ of $\mathfrak{s}$ given by

$$
\mathfrak{p}(\boldsymbol{s})=\min \left\{\boldsymbol{p} \in \mathbb{Z}^{c} \mid \mathfrak{s}(\boldsymbol{p}) \geq \boldsymbol{s}\right\} .
$$

In the $c$-tuple complex $\mathbb{P}_{1} \otimes_{k} \mathbb{P}_{2} \otimes_{k} \cdots \otimes_{k} \mathbb{P}_{c}$ all terms are of the form

$$
\Lambda_{1}^{\mathrm{en}}\left\langle s_{1}\right\rangle \otimes_{k} \cdots \otimes_{k} \Lambda_{c}^{\mathrm{en}}\left\langle s_{c}\right\rangle
$$

for some $\boldsymbol{s} \in \mathbb{N}^{c}$. We recall how these are identified with $\Lambda^{\mathrm{en}}\langle\boldsymbol{s}\rangle$.

Lemma 2.1 [Bergh and Oppermann 2008a, Lemma 4.3]. For $s \in \mathbb{Z}^{c}$ we choose an identification

$$
\Lambda_{1}^{\mathrm{en}}\left\langle s_{1}\right\rangle \otimes_{k} \cdots \otimes_{k} \Lambda_{c}^{\mathrm{en}}\left\langle s_{c}\right\rangle=\Lambda^{\mathrm{en}}\langle\boldsymbol{s}\rangle
$$

such that $(1 \otimes 1) \otimes \cdots \otimes(1 \otimes 1)$ maps to $1 \otimes 1$. Under such an identification,

$$
\left(x_{1}^{a_{1}} \otimes x_{1}^{b_{1}}\right) \otimes \cdots \otimes\left(x_{c}^{a_{c}} \otimes x_{c}^{b_{c}}\right) \quad \text { maps to } \quad \frac{\boldsymbol{q}^{\langle\boldsymbol{s} \mid \boldsymbol{s}\rangle}}{\boldsymbol{q}^{\langle\boldsymbol{a}+\boldsymbol{s} \mid \boldsymbol{b}+\boldsymbol{s}\rangle}} \boldsymbol{x}^{\boldsymbol{a}} \otimes \boldsymbol{x}^{\boldsymbol{b}} .
$$

Remark 2.2. The differentials occurring in the various directions of the $c$-tuple complex being of particular interest, we note that the identification of Lemma 2.1 maps $(1 \otimes 1) \otimes \cdots \otimes(1 \otimes 1) \otimes\left(x_{i} \otimes 1-1 \otimes x_{i}\right) \otimes(1 \otimes 1) \otimes \cdots \otimes(1 \otimes 1)$ to

$$
\frac{1}{\boldsymbol{q}^{\left\langle e_{i} \mid s\right\rangle}} x_{i} \otimes 1-\frac{1}{\boldsymbol{q}^{\left\langle s \mid e_{i}\right\rangle}} 1 \otimes x_{i}
$$


and $(1 \otimes 1) \otimes \cdots \otimes(1 \otimes 1) \otimes\left(\sum_{j=0}^{n_{i}-1} x_{i}^{j} \otimes x_{i}^{n_{i}-1-j}\right) \otimes(1 \otimes 1) \otimes \cdots \otimes(1 \otimes 1)$ to $\sum_{j=0}^{n_{i}-1} \frac{1}{\boldsymbol{q}^{\left\langle j e_{i} \mid \boldsymbol{s}\right\rangle} \boldsymbol{q}^{\left\langle\boldsymbol{s} \mid\left(n_{i}-1-j\right) e_{i}\right\rangle}} x_{i}^{j} \otimes x_{i}^{n_{i}-1-j}=\sum_{j=0}^{n_{i}-1}\left(\frac{1}{\boldsymbol{q}^{\left\langle e_{i} \mid \boldsymbol{s}\right\rangle}}\right)^{j}\left(\frac{1}{\boldsymbol{q}^{\left\langle\boldsymbol{s} \mid e_{i}\right\rangle}}\right)^{n_{i}-1-j} x_{i}^{j} \otimes x_{i}^{n_{i}-1-j}$.

Technical notation. We need some definitions to keep the notation short in the rest of the paper.

I. We set $Q=\left(q_{i j}\right)_{i j}$, and think of $Q$ as a (skew symmetric) matrix with entries in the abelian group $k^{\times}$. That is, $Q$ represents the morphism of abelian groups

$$
Q: \mathbb{Z}^{c} \rightarrow\left(k^{\times}\right)^{c}, \quad\left(d_{i}\right)_{i} \mapsto\left(\prod_{j=1}^{c} q_{i j}^{d_{j}}\right)_{i} .
$$

As usual for matrices we will denote the image of $\boldsymbol{d} \in \mathbb{Z}^{c}$ under this map by $Q d$, and its $i$-th component by $(Q d)_{i}$.

For $A, B \subseteq\{1, \ldots, c\}$, denote by $Q_{A \times B}$ the submatrix containing only the rows indexed by $A$ and the columns indexed by $B$ : that is, the matrix representing the composition

$$
\mathbb{Z}^{B C} \longrightarrow \mathbb{Z}^{c} \stackrel{Q}{\longrightarrow}\left(k^{\times}\right)^{c} \longrightarrow\left(k^{\times}\right)^{A} .
$$

II. We set $\mathscr{R}_{i}= \begin{cases}\left\{\zeta \mid \zeta^{n_{i}}=1\right\} & \text { if char } k \text { divides } n_{i}, \\ \left\{\zeta \mid \zeta^{n_{i}}=1 \text { and } \zeta \neq 1\right\} & \text { otherwise. }\end{cases}$

III. For a $\mathbb{Z}$-submodule $K$ of $\mathbb{Z}^{a}$, denote by pos.rk $K$ the rank of the $\mathbb{Z}$-submodule $K^{\prime}$ of $K$ generated by $K \cap \mathbb{N}^{a}$. For example,

$$
\text { pos.rk }\left\langle\left(\begin{array}{l}
1 \\
0 \\
0
\end{array}\right),\left(\begin{array}{r}
0 \\
1 \\
-1
\end{array}\right)\right\rangle=1
$$

\section{Hochschild cohomology}

For $\boldsymbol{d} \in \mathbb{Z}^{c}$, we will calculate the degree- $\boldsymbol{d}$ part of the Hochschild cohomology. Then we will obtain the entire Hochschild cohomology by adding up these parts.

To find the degree- $\boldsymbol{d}$ part of the cohomology we first have to understand the set

$$
\operatorname{Hom}_{\Lambda^{\mathrm{en}}}^{d}\left(\Lambda^{\mathrm{en}}\langle s\rangle, \Lambda\right)
$$

of degree- $\boldsymbol{d}$ morphisms from the terms of the projective resolution to $\Lambda$.

Lemma 3.1. The set $\operatorname{Hom}_{\Lambda^{\mathrm{en}}}^{\boldsymbol{d}}\left(\Lambda^{\mathrm{en}}\langle\boldsymbol{s}\rangle, \Lambda\right)$ is nonzero if and only if $\mathbf{0} \leq \boldsymbol{s}+\boldsymbol{d} \leq \boldsymbol{n}-\mathbf{1}$, and then it is the one-dimensional $k$-vector space generated by

$$
\varphi^{s, d}: \Lambda^{\mathrm{en}}\langle s\rangle \rightarrow \Lambda, \quad \boldsymbol{x}^{a} \otimes \boldsymbol{x}^{b} \mapsto q^{\langle a+s+d \mid b+s+d\rangle} x^{a+s+d+b} .
$$


Proof. Clearly any $\Lambda^{\text {en }}$-homomorphism from $\Lambda^{\text {en }}\langle\boldsymbol{s}\rangle$ to any other module is uniquely determined by the image of $1 \otimes 1$. If the morphism is to be of degree $\boldsymbol{d}$, this image can only be a scalar multiple of $\boldsymbol{x}^{\boldsymbol{s}+\boldsymbol{d}}$. We choose the image of $1 \otimes 1$ to be $\boldsymbol{q}^{\langle\boldsymbol{s}+\boldsymbol{d} \mid \boldsymbol{s}+\boldsymbol{d}\rangle} \boldsymbol{x}^{\boldsymbol{s}+\boldsymbol{d}}$ and obtain the formula of the lemma by extending $\Lambda^{\text {en }}$-linearly.

Corollary 3.2. $\operatorname{dim} \operatorname{Hom}_{\Lambda^{\mathrm{en}}}^{\boldsymbol{d}}\left(\Lambda^{\mathrm{en}}\langle\mathfrak{s}(\boldsymbol{p})\rangle, \Lambda\right)= \begin{cases}1 & \text { if } \mathfrak{p}(-\boldsymbol{d}) \leq \boldsymbol{p} \leq \mathfrak{p}(-\boldsymbol{d})+\mathbf{1}, \\ 0 & \text { otherwise. }\end{cases}$

This means that for $\boldsymbol{d} \leq \boldsymbol{n}-\mathbf{1}$ the $c$-tuple complex $\operatorname{Hom}_{\Lambda^{\mathrm{en}}}^{\boldsymbol{d}}\left(\mathbb{P}_{1} \otimes \cdots \otimes \mathbb{P}_{c}, \Lambda\right)$ is concentrated in a cube of sides 1 or 0 (the latter case occurring in directions $i$ with $\mathfrak{p}(-\boldsymbol{d})_{i}=-1$, i.e., $\left.d_{i}=n_{i}-1\right)$, where there is a one-dimensional space in each corner of the cube.

Since by Remark 2.2 these are the terms occurring in the projective resolution, we are in particular interested in what the maps $\varphi^{\boldsymbol{s}, \boldsymbol{d}}$ of Lemma 3.1 do to terms of the form

$\frac{1}{\boldsymbol{q}^{\left\langle e_{i} \mid s\right\rangle}} x_{i} \otimes 1-\frac{1}{\boldsymbol{q}^{\left\langle\boldsymbol{s} \mid e_{i}\right\rangle}} 1 \otimes x_{i} \quad$ and $\quad \sum_{j=0}^{n_{i}-1}\left(\frac{1}{\boldsymbol{q}^{\left\langle e_{i} \mid \boldsymbol{s}\right\rangle}}\right)^{j}\left(\frac{1}{\boldsymbol{q}^{\left\langle\boldsymbol{s} \mid e_{i}\right\rangle}}\right)^{n_{i}-1-j} x_{i}^{j} \otimes x_{i}^{n_{i}-1-j}$.

Lemma 3.3. Let $\boldsymbol{s}$ and $\boldsymbol{d}$ be such that $\mathbf{0} \leq \boldsymbol{s}+\boldsymbol{d} \leq \boldsymbol{n}-\mathbf{1}$, and let $i \in\{1 \ldots c\}$.

(1) Assume that $s_{i}+d_{i}+1<n_{i}$. Then

$$
\varphi^{s, d}\left(\frac{1}{\boldsymbol{q}^{\left\langle e_{i} \mid s\right\rangle}} x_{i} \otimes 1-\frac{1}{\boldsymbol{q}^{\left\langle\boldsymbol{s} \mid e_{i}\right\rangle}} 1 \otimes x_{i}\right)=0
$$

if and only if $(Q d)_{i}=1$. (For the definition of $Q$ see Technical notation I.)

(2) Assume that $s_{i}+d_{i}=0$. Then

$$
\varphi^{\boldsymbol{s}, \boldsymbol{d}}\left(\sum_{j=0}^{n_{i}-1}\left(\frac{1}{\boldsymbol{q}^{\left\langle e_{i} \mid \boldsymbol{s}\right\rangle}}\right)^{j}\left(\frac{1}{\boldsymbol{q}^{\left\langle\boldsymbol{s} \mid e_{i}\right\rangle}}\right)^{n_{i}-1-j} x_{i}^{j} \otimes x_{i}^{n_{i}-1-j}\right)=0
$$

if and only if $(Q d)_{i} \in \mathscr{R}_{i}$. (For the definition of $Q$ see Technical notation II.)

Proof. We only prove (2). The proof of (1) is a similar and simpler calculation using Lemma 3.1. By that lemma we have

$$
\begin{aligned}
\varphi^{\boldsymbol{s}, \boldsymbol{d}}\left(\sum_{j=0}^{n_{i}-1}\left(\frac{1}{\boldsymbol{q}^{\left\langle e_{i} \mid \boldsymbol{s}\right\rangle}}\right)^{j}\left(\frac{1}{\boldsymbol{q}^{\left\langle\boldsymbol{s} \mid e_{i}\right\rangle}}\right)^{n_{i}-1-j} x_{i}^{j} \otimes x_{i}^{n_{i}-1-j}\right) \\
\quad=\sum_{j=0}^{n_{i}-1}\left(\frac{1}{\boldsymbol{q}^{\left\langle e_{i} \mid \boldsymbol{s}\right\rangle}}\right)^{j}\left(\frac{1}{\boldsymbol{q}^{\left\langle\boldsymbol{s} \mid e_{i}\right\rangle}}\right)^{n_{i}-1-j} \boldsymbol{q}^{\left\langle j e_{i}+\boldsymbol{s}+\boldsymbol{d} \mid\left(n_{i}-1-j\right) e_{i}+\boldsymbol{s}+\boldsymbol{d}\right\rangle} \boldsymbol{x}^{\boldsymbol{s}+\boldsymbol{d}+\left(n_{i}-1\right) e_{i}} .
\end{aligned}
$$


Upon rearrangement of the right-hand side this becomes

$$
\begin{aligned}
& \boldsymbol{q}^{\langle\boldsymbol{s}+\boldsymbol{d} \mid \boldsymbol{s}+\boldsymbol{d}\rangle} \sum_{j=0}^{n_{i}-1}\left(\boldsymbol{q}^{\left\langle e_{i} \mid \boldsymbol{d}\right\rangle}\right)^{j}\left(\boldsymbol{q}^{\left\langle\boldsymbol{d} \mid e_{i}\right\rangle}\right)^{n_{i}-1-j} \boldsymbol{x}^{\boldsymbol{s}+\boldsymbol{d}+\left(n_{i}-1\right) e_{i}} \\
& =\underbrace{\boldsymbol{q}^{\langle\boldsymbol{s}+\boldsymbol{d} \mid \boldsymbol{s}+\boldsymbol{d}\rangle}}_{\neq 0} \boldsymbol{x}^{\boldsymbol{s}+\boldsymbol{d}+\left(n_{i}-1\right) e_{i}} \cdot \begin{cases}n_{i} \overbrace{\left(\boldsymbol{q}^{\left\langle e_{i} \mid \boldsymbol{d}\right\rangle}\right)^{n_{i}-1}} & \text { if } \boldsymbol{q}^{\left\langle e_{i} \mid \boldsymbol{d}\right\rangle}=\boldsymbol{q}^{\left\langle\boldsymbol{d} \mid e_{i}\right\rangle}, \\
\frac{\left(\boldsymbol{q}^{\left\langle e_{i} \mid \boldsymbol{d}\right\rangle}\right)^{n_{i}}-\left(\boldsymbol{q}^{\left\langle\boldsymbol{d} \mid e_{i}\right\rangle}\right)^{n_{i}}}{\boldsymbol{q}^{\left\langle e_{i} \mid \boldsymbol{d}\right\rangle}-\boldsymbol{q}^{\left\langle\boldsymbol{d} \mid e_{i}\right\rangle}} & \text { otherwise. }\end{cases}
\end{aligned}
$$

Now the claim follows from the fact that $\frac{\boldsymbol{q}^{\left\langle\boldsymbol{d} \mid e_{i}\right\rangle}}{\boldsymbol{q}^{\left\langle e_{i} \mid \boldsymbol{d}\right\rangle}}=\prod_{j=1}^{c} q_{i j}^{d_{j}}=(Q \boldsymbol{d})_{i}$.

We have shown that the vanishing of the maps on the edges in direction $i$ of the cube $\operatorname{Hom}_{\Lambda^{\text {en }}}\left(\mathbb{P}_{1} \otimes \cdots \otimes \mathbb{P}_{c}, \Lambda\right)$ does not depend on $s$, that is, if one edge in direction $i$ vanishes, then all vanish. Also, if all the edges in one direction are isomorphisms, the total complex is acyclic. Hence, if we partition the set $\{1, \ldots, c\}$ into subsets

$$
\begin{aligned}
I_{\max } & =\left\{i \in\{1, \ldots, c\}: n_{i}=d_{i}+1\right\}, \\
I_{1} & =\left\{i \in\{1, \ldots, c\}: n_{i} \mid d_{i}+1\right\} \backslash I_{\max }, \\
I_{2} & =\left\{i \in\{1, \ldots, c\}: n_{i} \nmid d_{i}+1\right\},
\end{aligned}
$$

we have shown the following:

Theorem 3.4. Let $\Lambda=\Lambda_{\boldsymbol{q}}^{\boldsymbol{n}}$ be a quantum complete intersection, and let $\boldsymbol{d} \leq \boldsymbol{n}-\mathbf{1}$. Then $\mathrm{HH}^{*, d}(\Lambda) \neq 0$ if and only if

- $(Q d)_{i} \in \mathscr{R}_{i}$ for all $i \in I_{1}$, and

- $(Q d)_{i}=1$ for all $i \in I_{2}$.

In this situation $\mathrm{HH}^{*, d}(\Lambda)$ has the $k$-vector space basis

$$
\left\{E_{\boldsymbol{p}}^{\boldsymbol{d}} \mid \mathbf{0} \leq \boldsymbol{p} \text { and } \mathfrak{p}(-\boldsymbol{d}) \leq \boldsymbol{p} \leq \mathfrak{p}(-\boldsymbol{d})+\mathbf{1}\right\},
$$

where $E_{p}^{d}$ is represented by the (degree-d) map from the c-tuple complex

$$
\mathbb{P}_{1} \otimes \cdots \otimes \mathbb{P}_{c}
$$

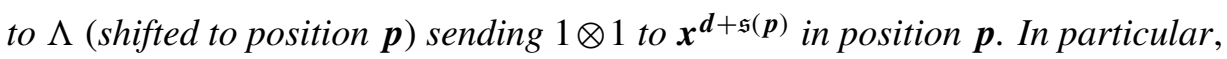

$$
E_{\boldsymbol{d}}^{\boldsymbol{p}} \text { has extension degree } \sum_{i=1}^{c} p_{i} \text {. }
$$

The assumptions on $\boldsymbol{p}$ in $(*)$ just ensure that $\mathbf{0} \leq \boldsymbol{d}+\mathfrak{s}(\boldsymbol{p}) \leq \boldsymbol{n}-\mathbf{1}$ : in other words, we are in the cube where $\operatorname{Hom}_{\Lambda^{\text {en }}}^{d}\left(\mathbb{P}_{1} \otimes \cdots \otimes \mathbb{P}_{c}, \Lambda\right)$ does not vanish. 
Now let us compare this result to the description of $\operatorname{Ext}_{\Lambda}^{*}(k, k)$ obtained in [Bergh and Oppermann 2008a]. More precisely: tensoring over $\Lambda$ with $k$ yields a map from the Hochschild cohomology to the Ext-algebra of the $\Lambda$-module $k$. Our aim now is to determine its image. By Theorem 5.3 of that reference, the latter ring has the form

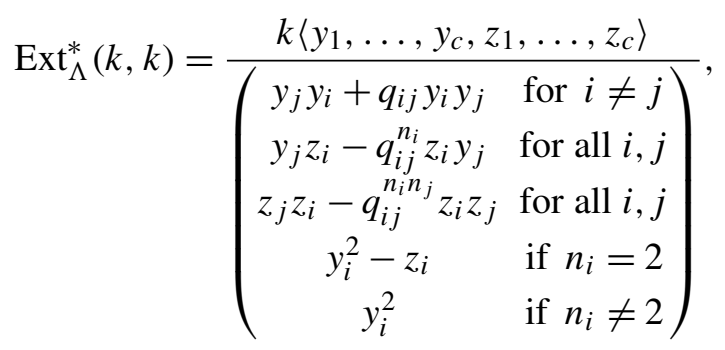

where $\left|y_{i}\right|=\left(1,-e_{i}\right)$ and $\left|z_{i}\right|=\left(2,-n_{i} e_{i}\right)$, and where the quotient is by the ideal generated by the polynomials indicated.

Corollary 3.5. The image of the map $\left(-\otimes_{\Lambda} k\right)_{*}: \mathrm{HH}^{*}(\Lambda) \rightarrow \operatorname{Ext}_{\Lambda}^{*}(k, k)$ is

$$
\bigoplus_{\boldsymbol{d} \in \mathscr{D}} \operatorname{Ext}^{*, \boldsymbol{d}}(k, k)
$$

where the sum runs over graded pieces where the corresponding graded piece of the Hochschild cohomology does not vanish:

$$
\mathscr{D}=\left\{\boldsymbol{d} \in \mathbb{Z}^{c}:(Q \boldsymbol{d})_{i} \in \mathscr{R}_{i} \text { for } i \in I_{\max } \cup I_{1},(Q \boldsymbol{d})_{i}=1 \text { for } i \in I_{2}\right\} .
$$

Proof. By construction the image cannot be bigger than the sum in $(\dagger)$. To see that any $\operatorname{Ext}^{*, \boldsymbol{d}}(k, k)$ with $\boldsymbol{d} \in \mathscr{D}$ is contained in the image, first note that

$$
\operatorname{dim}_{k} \operatorname{Ext}^{*, \boldsymbol{d}}(k, k)= \begin{cases}1 & \text { if } \forall i: d_{i} \leq 0 \text { and } n_{i}\left|d_{i} \vee n_{i}\right| d_{i}+1 \\ 0 & \text { otherwise }\end{cases}
$$

The condition for $\operatorname{Ext}^{*, \boldsymbol{d}}(k, k)$ not vanishing is equivalent to asking that $\boldsymbol{d}=-\mathfrak{s}(\boldsymbol{p})$ for some $\boldsymbol{p} \in \mathbb{N}^{c}$. By definition, $E_{p}^{d}$ is represented by a map sending $1 \otimes 1$ to 1 in position $\boldsymbol{p}$, and hence it does not vanish when being tensored over $\Lambda$ by $k$. Therefore the image is at least one-dimensional in degree $\boldsymbol{d}$.

\section{The rate of growth of the Hochschild cohomology}

In this section we study how big the Hochschild cohomology of a finite-dimensional quantum complete intersection is. Our way to measure for measuring the size is the rate of growth, as explained in the following definition. 
Definition 4.1. Let $X=\bigsqcup_{i=0}^{\infty} X_{i}$ be an $\mathbb{N}$-graded $k$-module such that the $X_{i}$ have finite $k$-dimension. The rate of growth of $X$, denoted by $\gamma(X)$, is defined as

$$
\gamma(X)=\inf \left\{t \in \mathbb{N} \mid \exists a \in \mathbb{N} \text { such that } \forall i: \operatorname{dim}_{k} X_{i} \leq a i^{t}\right\} .
$$

If $X$ is a graded commutative ring that is finitely generated over $k$, then $\gamma(X)=$ Krull.dim $X$. However this assumption is not always satisfied for the Hochschild cohomology ring of quantum complete intersections (see Sections 5 and 6).

We first decompose the Hochschild cohomology as follows:

Construction 4.2. For $G \subseteq\{1, \ldots, c\}$ we denote by $\mathrm{HH}_{G}^{*}$ the $k$-span of the $E_{p}^{d}$ for which $\boldsymbol{d}$ and $\boldsymbol{n}$ satisfy the condition

$$
G=\left\{i \in\{1, \ldots, c\} \mid d_{i}<n_{i}-1 \text { or }(Q d)_{i} \in \mathscr{R}_{i}\right\} .
$$

That is, we take all those $E_{\boldsymbol{p}}^{\boldsymbol{d}}$ from Theorem 3.4 such that $G$ contains exactly the indices not in $I_{\max }$ plus those in $I_{\max }$ that fulfill the requirements for elements of $I_{1}$.

Clearly this yields a decomposition $\mathrm{HH}^{*}(\Lambda)=\bigoplus_{G \subseteq\{1, \ldots, c\} \mathrm{HH}_{G}^{*}}$, and hence

$$
\gamma\left(\mathrm{HH}^{*}(\Lambda)\right)=\max _{G \subseteq\{1, \ldots, c\}} \gamma\left(\mathrm{HH}_{G}^{*}\right) .
$$

Proposition 4.3. For $G \subseteq\{1, \ldots, c\}$ the rate of growth of $\mathrm{HH}_{G}^{*}$ is

$$
\gamma\left(\mathrm{HH}_{G}^{*}\right)= \begin{cases}0 & \text { if } \mathrm{HH}_{G}^{*}=0, \\ \text { pos.rk Ker } Q_{G \times G} & \text { otherwise. }\end{cases}
$$

(For the definition of pos.rk see Technical notation III.) In particular we always have $\gamma\left(\mathrm{HH}_{G}^{*}\right) \leq|G|$.

For the proof we will need the following observation.

Observation 4.4. Let $K \leq \mathbb{Z}^{a}$ be a submodule. The k-module with basis $K \cap \mathbb{N}^{a}$ is $\mathbb{Z}$-graded by $|\boldsymbol{x}|=\sum_{i=1}^{a} x_{i}$ for $\boldsymbol{x} \in K$. With this grading, its rate of growth is $\gamma\left(k\left(K \cap \mathbb{N}^{a}\right)\right)=$ pos.rk $K$.

Proof of Proposition 4.3. By construction, $\mathrm{HH}_{G}^{*}$ has the $k$-basis

$$
\begin{aligned}
& \left\{E_{\boldsymbol{p}}^{\boldsymbol{d}} \mid \boldsymbol{p} \geq \mathbf{0}, \boldsymbol{d} \leq \boldsymbol{n}-\mathbf{1}, \mathfrak{p}(-\boldsymbol{d}) \leq \boldsymbol{p} \leq \mathfrak{p}(-\boldsymbol{d})+\mathbf{1}, d_{i}=n_{i}-1 \text { for } i \notin G,\right. \\
& \left.(Q \boldsymbol{d})_{i} \notin \mathscr{R}_{i} \text { for } i \notin G,(Q \boldsymbol{d})_{i} \in \mathscr{R}_{i} \text { for } i \in G \backslash I_{2},(Q \boldsymbol{d})_{i}=1 \text { for } i \in G \cap I_{2}\right\},
\end{aligned}
$$

and the extension degree of $E_{\boldsymbol{p}}^{\boldsymbol{d}}$ is $\sum_{i=1}^{c} p_{i}$.

Note that the map $\mathfrak{p}$ is linear up to some rounding. Hence we may calculate the rate of growth with respect to the grading given by $-\sum_{i=1}^{c} d_{i}$.

Since for any $\boldsymbol{d}$ there are at least one and at most $2^{c}$ values of $\boldsymbol{p}$ satisfying the conditions of the set above, we may disregard the number of different choices for $\boldsymbol{p}$ for a given $\boldsymbol{d}$. 
Next, since $\boldsymbol{d}$ is fixed outside $G$, we may restrict our attention to the $G$ part of the indices. Write $\bar{G}=\{1, \ldots, c\} \backslash G$ and define, for $G \subseteq G^{\prime} \subseteq\{1, \ldots, c\}$ the set

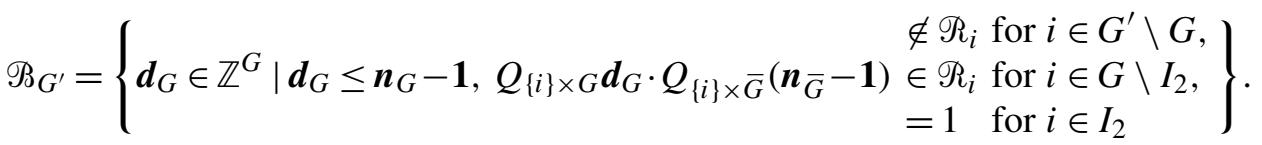

We need to understand the rate of growth of the $k$-module with basis $\mathscr{B}_{\{1, \ldots, c\}}$. Note that $\mathscr{B}_{\{1, \ldots, c\}} \subseteq \mathscr{B}_{G}$ (more generally, for $G^{\prime} \subseteq G^{\prime \prime}$ we have $\mathscr{B}_{G^{\prime}} \supseteq \mathscr{B}_{G^{\prime \prime}}$ ).

Now $\mathscr{B}_{G}$ is invariant under adding elements of the set

$$
-\left(\prod_{i \in G} n_{i} \mathbb{N}\right) \cap \operatorname{Ker} Q_{G \times G},
$$

and contains only finitely many elements which are not obtained from another element by such an addition. Hence, if $\mathscr{B}_{G}$ is nonempty, the rate of growth of the $k$-module with basis $\mathscr{B}_{G}$ is identical to the rate of growth of the $k$-module with basis $\mathbb{N}^{G} \cap \operatorname{Ker} Q_{G \times G}$, which, by Observation 4.4, is pos.rk $\operatorname{Ker} Q_{G \times G}$.

It follows that $\gamma\left(\mathrm{HH}_{G}^{*}\right) \leq$ pos.rk $\operatorname{Ker} Q_{G \times G}$.

Now we let $\widehat{G}$ be maximal with $G \subseteq \widehat{G} \subseteq\{1, \ldots, c\}$ such that pos.rk Ker $Q_{\widehat{G} \times G}=$ pos.rk Ker $Q_{G \times G}$. It follows, as in the discussion above, that if $\mathscr{B}_{\widehat{G}} \neq \varnothing$ then the rate of growth of the $k$-module with basis $\mathscr{B}_{\widehat{G}}$ is pos.rk $\operatorname{Ker} Q_{G \times G}$.

Finally let $i \notin \widehat{G}$. Using arguments similar to the foregoing, one sees that the rate of growth of the free module with basis $\mathscr{B}_{G} \backslash \mathscr{B}_{G \cup\{i\}}$ is strictly smaller than pos.rk $\operatorname{Ker} Q_{G \times G}$.

Since

$$
\mathscr{B}_{\{1, \ldots, c\}}=\mathscr{B}_{\widehat{G}} \backslash\left(\bigcup_{i \notin \widehat{G}}\left(\mathscr{R}_{G} \backslash \mathscr{P}_{G \cup\{i\}}\right)\right),
$$

it follows that, provided $\mathscr{B}_{\{1, \ldots, c\}} \neq \varnothing$, the rate of growth of the $k$-module with basis $\mathscr{B}_{\{1, \ldots, c\}}$ is pos.rk $\operatorname{Ker} Q_{G \times G}$.

Summing up the results for $\mathrm{HH}_{G}^{*}$, we have shown:

Theorem 4.5. The rate of growth of the Hochschild cohomology of a finite-dimensional quantum complete intersection is the maximum of pos.rk $\operatorname{Ker} Q_{G \times G}$ over

$$
G=\left\{i \in\{1, \ldots, c\} \mid d_{i}<n_{i}-1 \text { or }(Q d)_{i} \in \mathscr{R}_{i}\right\},
$$

where $\boldsymbol{d}$ ranges over elements of $\mathbb{Z}^{c}$ such that $\boldsymbol{d} \leq \boldsymbol{n}-\mathbf{1},(Q \boldsymbol{d})_{i} \in \mathscr{R}_{i}$ for all $\boldsymbol{i}$ with $n_{i} \mid d_{i}+1$ and $d_{i}<0$, and $(Q \boldsymbol{d})_{i}=1$ for all $i$ with $n_{i} \nmid d_{i}+1$.

Corollary 4.6. For a finite quantum complete intersection either all $q_{i j}$ are roots of unity, or the rate of growth of the Hochschild cohomology is at most c -2 .

Proof. Assume not all $q_{i j}$ are roots of unity. Then rk Ker $Q \leq c-2$, since $Q$ is skew symmetric. Hence pos.rk $\operatorname{Ker} Q \leq c-2$. Now we consider $G$ with $|G|=c-1$, 
that is, $G=\{1, \ldots, c\} \backslash\{h\}$ for some $h$. If rk Ker $Q_{G \times G} \leq c-2$ there is nothing to show, so assume $Q_{G \times G}$ only contains roots of unity. Since $Q$ does not only contain roots of unity there is $i \in G$ such that $q_{i h}$ is not a root of unity. But then $(Q \boldsymbol{d})_{i}$ cannot be a root of unity for any $\boldsymbol{d} \in \mathbb{Z}^{c}$ with $d_{h}=n_{h}-1 \neq 0$. Hence this $G$ is not to be considered in the maximum of Theorem 4.5.

\section{On the multiplicative structure of the Hochschild cohomology}

In this section we will identify a subring $\mathscr{S}$ of the Hochschild cohomology ring, which is a finitely generated commutative $k$-algebra without zero divisors, and is isomorphic to the Hochschild cohomology modulo nilpotent objects. We will completely describe $\mathscr{Y}$, determine its Krull dimension, and determine when the entire Hochschild cohomology ring is finitely generated as a module over $\mathscr{Y}$.

By Theorem 3.4 we know that the Hochschild cohomology has a $k$-vector space basis

$$
\begin{aligned}
& \left\{E_{\boldsymbol{p}}^{\boldsymbol{d}} \mid \boldsymbol{p} \geq \mathbf{0}, \mathfrak{p}(-\boldsymbol{d}) \leq \boldsymbol{p} \leq \mathfrak{p}(-\boldsymbol{d})+\mathbf{1},\right. \\
& \left.\quad(Q \boldsymbol{d})_{i} \in \mathscr{R}_{i} \text { for all } i \text { with } n_{i} \mid d_{i}+1>0,(Q \boldsymbol{d})_{i}=1 \text { for all } i \text { with } n_{i} \nmid d_{i}+1 .\right\}
\end{aligned}
$$

For simplicity of notation we set $E_{\boldsymbol{p}}^{\boldsymbol{d}}=0$ whenever $\boldsymbol{d}$ and $\boldsymbol{p}$ do not satisfy the conditions above. Then we always have

$$
E_{\boldsymbol{p}}^{\boldsymbol{d}} \cdot E_{\boldsymbol{p}^{\prime}}^{\boldsymbol{d}^{\prime} \in k E_{\boldsymbol{p}+\boldsymbol{p}^{\prime}}^{\boldsymbol{d}+\boldsymbol{d}^{\prime}}}
$$

Lemma 5.1. Assume $\mathfrak{s}(\boldsymbol{p}) \neq-\boldsymbol{d}$. Then $E_{\boldsymbol{p}}^{\boldsymbol{d}}$ is nilpotent.

Proof. Let $i$ be such that $\mathfrak{s}(\boldsymbol{p})_{i}>-d_{i}$. Then

$$
\mathfrak{s}\left(n_{i} \boldsymbol{p}\right)_{i} \geq n_{i} \mathfrak{s}(\boldsymbol{p})_{i} \geq n_{i}\left(1-d_{i}\right) \geq n_{i}-n_{i} d_{i},
$$

and hence $\left(E_{p}^{\boldsymbol{d}}\right)^{n_{i}} \in k E_{n_{i} p}^{n_{i} \boldsymbol{d}}=0$.

We are particularly interested in the nonnilpotent elements of the Hochschild cohomology ring. For simplicity of notation, we give the remaining candidates a new name:

$$
s_{\boldsymbol{p}}:=E_{\boldsymbol{p}}^{-\mathfrak{s}(\boldsymbol{p})} .
$$

Lemma 5.2. Let $\boldsymbol{p} \in \mathbb{N}^{c}$ such that there is $i \in\{1, \ldots, c\}$ with $n_{i}>2$ and $p_{i}$ is odd. Then $s_{p}$ is nilpotent.

Proof. A straightforward calculation shows that $\left(s_{p}\right)^{2}$ satisfies the assumption of Lemma 5.1.

Now we set

$$
\begin{aligned}
\mathscr{S}={ }_{k}\left\langle s_{\boldsymbol{p}}\right|(Q \mathfrak{s}(\boldsymbol{p}))_{i} & =1 \text { for all } i \text { with } p_{i} \text { even, } \\
(Q \mathfrak{s}(\boldsymbol{p}))_{i} & \left.=-1 \text { and } n_{i}=2 \text { for all } i \text { with } p_{i} \text { odd }\right\rangle .
\end{aligned}
$$


By the preceding two lemmas, the composition $\mathscr{S} \hookrightarrow \mathrm{HH}^{*}(\Lambda) \rightarrow \frac{\mathrm{HH}^{*}(\Lambda)}{\text { (nilpotence) }}$ is onto.

Our next aim is to understand how the elements of $\mathscr{Y}$ are multiplied with each other and with the other $E_{\boldsymbol{p}}^{\boldsymbol{d}}$. To do so we lift the map representing $s_{\boldsymbol{p}}$, with $\boldsymbol{p}$ as in the definition of $\mathscr{Y}$, to a map of $c$-tuple complexes.

Lemma 5.3. The element $s_{p}$ with $\boldsymbol{p}$ as in the definition of $\mathscr{Y}$ is represented by the map of c-tuple complexes $\mathbb{P}_{1} \otimes \cdots \otimes \mathbb{P}_{c} \rightarrow\left(\mathbb{P}_{1} \otimes \cdots \otimes \mathbb{P}_{c}\right)[\boldsymbol{p}]$ that sends $1 \otimes 1$ to $\left(1 / \boldsymbol{q}^{\langle\mathfrak{s}(\boldsymbol{r}) \mid \mathfrak{s}(\boldsymbol{p})\rangle}\right) \cdot 1 \otimes 1$ in position $\boldsymbol{p}+\boldsymbol{r}$.

Proof. It suffices to verify that the map given in the lemma is a map of $c$-tuple complexes, since then it clearly does the right thing in position $\boldsymbol{p}$. This amounts to checking that the various squares commute - a straightforward, if somewhat tiresome, calculation involving four different cases, according to the parities of the $p_{i}$ and $r_{i}$.

Note that when passing from $c$-tuple complexes to their total complexes some maps need to be multiplied by -1 . One choice for doing so is to multiply the map in direction $i$ from position $\boldsymbol{p}+e_{i}$ to $\boldsymbol{p}$ by $\prod_{j<i}(-1)^{p_{i}}$. With this convention we have the following immediate consequence of Lemma 5.3.

Corollary 5.4. We have $s_{\boldsymbol{p}} E_{\boldsymbol{p}^{\prime}}^{\boldsymbol{d}}=\frac{\prod_{j<i}(-1)^{p_{j} p_{i}^{\prime}}}{\boldsymbol{q}^{\left\langle\mathfrak{s}\left(\boldsymbol{p}^{\prime}\right) \mid \mathfrak{s}(\boldsymbol{p})\right\rangle}} E_{\boldsymbol{p}+\boldsymbol{p}^{\prime}}^{\boldsymbol{d}-\mathfrak{s}(\boldsymbol{p})}$. In particular,

$$
s_{\boldsymbol{p}} s_{\boldsymbol{p}^{\prime}}=\frac{\prod_{j<i}(-1)^{p_{j} p_{i}^{\prime}}}{\boldsymbol{q}^{\left\langle\mathfrak{s}\left(\boldsymbol{p}^{\prime}\right) \mid \mathfrak{s}(\boldsymbol{p})\right\rangle}} s_{\boldsymbol{p}+\boldsymbol{p}^{\prime}} .
$$

From these results we obtain:

Theorem 5.5. The Hochschild cohomology ring of a quantum complete intersection has a subring $\mathscr{Y}$ isomorphic to ${ }_{k}\left\langle y_{1}^{p_{1} n_{1} / 2} \ldots y_{c}^{p_{c} n_{c} / 2} \in k\left[y_{1}, \ldots y_{c}\right]\right|(Q \mathfrak{s}(\boldsymbol{p}))_{i}=1$ for all $i$ with $p_{i}$ even,

$$
\left.(Q \mathfrak{s}(\boldsymbol{p}))_{i}=-1 \text { and } n_{i}=2 \text { for all } i \text { with } p_{i} \text { odd }\right\rangle .
$$

In particular, $\mathscr{Y}$ is a finitely generated $k$-algebra without zero-divisors.

Moreover the composition

$$
\mathscr{S} \hookrightarrow \mathrm{HH}^{*}(\Lambda) \rightarrow \frac{\mathrm{HH}^{*}(\Lambda)}{(\text { nilpotence) }}
$$

is an isomorphism. Hence $\frac{\mathrm{HH}^{*}(\Lambda)}{(\text { nilpotence) }}$ is a split quotient of $\mathrm{HH}^{*}(\Lambda)$ and is isomorphic to 9 .

Proof. That the $s_{p}$ commute can be checked directly, using ( $\ddagger$ ) in Corollary 5.4. Alternatively, note that, since $(\ddagger)$ implies $s_{\boldsymbol{p}}^{2} \neq 0$, either $s_{\boldsymbol{p}}$ lies in the even part of 
the Hochschild cohomology or char $k=2$. In both cases it follows from general theory that $s_{p}$ lies in the center of the Hochschild cohomology ring.

Thus $\mathscr{S}$ has the form described in the theorem.

To see that $\mathscr{Y}$ is finitely generated as a $k$-algebra we partially order the set $\left\{y_{1}^{p_{1} n_{1} / 2} \ldots y_{c}^{p_{c} n_{c} / 2} \in \mathscr{S}\right\}$ by comparing the exponents componentwise. Since the ideal in $k\left[y_{1}, \ldots, y_{c}\right]$ generated by this set is finitely generated, it follows there are only finitely many minimal elements with respect to this partial order. We claim that they generate $\mathscr{S}$ as a $k$-algebra. Assume that $y_{1}^{p_{1} n_{1} / 2} \ldots y_{c}^{p_{c} n_{c} / 2} \in \mathscr{S}$ is not minimal. Then one easily sees that $y_{1}^{p_{1} n_{1} / 2} \ldots y_{c}^{p_{c} n_{c} / 2}$ is the product of two smaller elements of this form (for instance, one of them could be chosen minimal). Iterating we see that any $y_{1}^{p_{1} n_{1} / 2} \ldots y_{c}^{p_{c} n_{c} / 2} \in \mathscr{Y}$ is a product of minimal ones.

The final part of the theorem follows from the comment at the top of page 832 and from Corollary 5.4.

We conclude this section by giving a precise criterion for when the entire Hochschild cohomology ring is finitely generated over $\mathscr{S}$.

Lemma 5.6. The decomposition $\mathrm{HH}^{*}(\Lambda)=\underset{G \subseteq\{1, \ldots, c\}}{\bigoplus} \mathrm{HH}_{G}^{*}$ of Construction 4.2
respects the $\mathscr{S}$-module structure. Proof. This follows immediately from the definition of $\mathrm{HH}_{G}^{*}$ and the multiplication formula in Corollary 5.4.

Proposition 5.7. The module $\mathrm{HH}_{G}^{*}$ is finitely generated over $\mathscr{Y}$ if and only if it is zero or pos.rk Ker $Q_{G \times G}=$ pos.rk $\operatorname{Ker} Q_{\{1, \ldots, c\} \times G}$.

Proof. Clearly we may assume $\mathrm{HH}_{G}^{*} \neq 0$. Note that the $s_{p}$ with $p_{i} \neq 0$ for some $i \in\{1, \ldots, c\} \backslash G$ annihilate $\mathrm{HH}_{G}^{*}$, and hence $\mathrm{HH}_{G}^{*} \neq 0$ is actually a module over the split quotient $\mathscr{S}_{G}:={ }_{k}\left\langle s_{\boldsymbol{p}} \in \mathscr{S} \mid \forall i: i \in G \vee p_{i}=0\right\rangle$. By Observation 4.4, $\gamma\left(\mathscr{S}_{G}\right)=$ pos.rk $\operatorname{Ker} Q_{\{1, \ldots, c\} \times G}$.

Moreover $\mathscr{Y}_{G}$ acts on $\mathrm{HH}_{G}^{*}$ without zero-divisors: Since both $\mathscr{Y}_{G}$ and $\mathrm{HH}_{G}^{*}$ are $\mathbb{Z}^{c}$-graded it suffices to look at graded parts. For these, this is immediate from the multiplication formula in Corollary 5.4. The claim follows.

Corollary 5.8. For any finite-dimensional quantum complete intersection $\mathrm{HH}_{\{1, \ldots, c\}}^{*}$ is a finitely generated $\mathscr{S}$-module.

Theorem 5.9. The Hochschild cohomology ring is finitely generated as a module over $\mathscr{S}$ if and only if pos.rk $\operatorname{Ker} Q_{G \times G}=$ pos.rk $\operatorname{Ker} Q_{\{1, \ldots, c\} \times G}$ for any subset $G \subseteq\{1, \ldots, c\}$ for which there exists $\boldsymbol{d} \in \mathbb{Z}^{c}$ satisfying

- $d \leq n-1$,

- $d_{i}=n_{i}-1$ for all $i \in\{1, \ldots, c\} \backslash G$,

- $(Q d)_{i} \in \mathscr{R}_{i}$ for all $i \in G$ with $n_{i} \mid d_{i}+1$,

- $(Q d)_{i}=1$ for all $i \in G$ with $n_{i} \nmid d_{i}+1$. 


\section{Examples}

Example 6.1 [Bergh and Erdmann 2008]. Let $\Lambda=\Lambda_{q_{12}}^{n_{1}, n_{2}}$ be a codimension-2 quantum complete intersection such that $q_{12}$ is not a root of unity. Take $\boldsymbol{d}=$ $\left(d_{1}, d_{2}\right) \leq\left(n_{1}-1, n_{2}-1\right)$. Then $\mathrm{HH}^{*, \boldsymbol{d}}(\Lambda)$ does not vanish if and only if for any $i \in\{1,2\}$ we have $d_{i}=n_{i}-1$ or $n_{i} \nmid d_{i}+1$ and $q_{12}^{d_{3-i}}=1$. Since $q_{12}$ is not a root of unity this means that for any $i$ with $d_{i}<n_{i}-1$ we have $d_{3-i}=0$. Therefore the only $\boldsymbol{d}$ that contribute to the Hochschild cohomology are $\left(n_{1}-1, n_{2}-1\right)$ and $(0,0)$. For $\boldsymbol{d}=\left(n_{1}-1, n_{2}-1\right)$ we obtain $I_{\max }=\{1,2\}, I_{1}=\varnothing, I_{2}=\varnothing$, and $\mathfrak{p}(-\boldsymbol{d})=(-1,-1)$. Hence

$$
\mathrm{HH}^{*, \boldsymbol{d}}(\Lambda)={ }_{k}\left\langle E_{(0,0)}^{\left(n_{1}-1, n_{2}-1\right)}\right\rangle .
$$

For $\boldsymbol{d}=(0,0)$ we obtain $I_{\max }=\varnothing, I_{1}=\varnothing, I_{2}=\{1,2\}$, and $\mathfrak{p}(-\boldsymbol{d})=(0,0)$. Hence

$$
\mathrm{HH}^{*, \boldsymbol{d}}(\Lambda)={ }_{k}\left\langle E_{(0,0)}^{(0,0)}, E_{(0,1)}^{(0,0)}, E_{(1,0)}^{(0,0)}, E_{(1,1)}^{(0,0)}\right\rangle .
$$

Summing up we obtain

$$
\mathrm{HH}^{*, \boldsymbol{d}}(\Lambda)={ }_{k}\left\langle E_{(0,0)}^{\left(n_{1}-1, n_{2}-1\right)}, E_{(0,0)}^{(0,0)}, E_{(0,1)}^{(0,0)}, E_{(1,0)}^{(0,0)}, E_{(1,1)}^{(0,0)}\right\rangle,
$$

and hence

$$
\operatorname{dim} \mathrm{HH}^{*}(\Lambda)=(2,2,1,0, \ldots)
$$

We generalize this example to arbitrary codimensions:

Example 6.2. Let $c \geq 2$ and assume the $q_{i j}$ are generic, meaning that $(Q d)_{i}$ is a root of unity only if $d_{j}=0$ for all $j \neq i$. Then $\mathrm{HH}^{*, \boldsymbol{d}}(\Lambda) \neq 0$ only for $\boldsymbol{d}=\boldsymbol{n}-\mathbf{1}$ or $\boldsymbol{d}=\mathbf{0}$. Similarly to Example 6.1 we obtain

$$
\mathrm{HH}^{*, \boldsymbol{n}-1}(\Lambda)={ }_{k}\left\langle E_{\mathbf{0}}^{\boldsymbol{n}-\mathbf{1}}\right\rangle, \quad \mathrm{HH}^{*, \mathbf{0}}(\Lambda)={ }_{k}\left\langle E_{\boldsymbol{p}}^{\mathbf{0}} \mid \mathbf{0} \leq \boldsymbol{p} \leq \mathbf{1}\right\rangle .
$$

In particular,

$$
\operatorname{dim} \mathrm{HH}^{*}(\Lambda)=\left(1+\left(\begin{array}{l}
c \\
0
\end{array}\right),\left(\begin{array}{l}
c \\
1
\end{array}\right),\left(\begin{array}{l}
c \\
2
\end{array}\right),\left(\begin{array}{l}
c \\
c
\end{array}\right), \ldots\right) .
$$

Since the total dimension is finite, the rate of growth $\gamma\left(\mathrm{HH}^{*}(\Lambda)\right)$ is 0 and $\mathscr{S}=k$.

Now let us look at the other extreme case.

Example 6.3 [Bergh and Oppermann 2008a]. Let $c \geq 2$ and let all $q_{i j}$ be roots of unity. Then

$$
\text { pos.rk Ker } Q_{G^{\prime} \times G}=\operatorname{rk} \operatorname{Ker} Q_{G^{\prime} \times G}=|G|
$$

for any $G, G^{\prime} \subseteq\{1, \ldots, c\}$. Hence $\operatorname{HH}^{*}(\Lambda)$ is finitely generated over $\mathscr{Y}$, and

$$
\text { Krull.dim } \mathscr{S}=\gamma\left(\mathrm{HH}^{*}(\Lambda)\right)=c .
$$

The final two examples illustrate that when $\gamma\left(\mathrm{HH}^{*}(\Lambda)\right)=c-2$ very different kinds of behavior can occur. 
Example 6.4. Let $q \in k^{\times}$not be a root of unity and let $c \in \mathbb{N}_{\geq 3}$. Let $\Lambda$ be a quantum complete intersection of codimension $c$, with

$$
\begin{array}{lll}
q_{i j}=1 & \text { for } i, j<c-1, & q_{i, c-1}=q \quad \text { for } i<c-1, \\
q_{i c}=q^{-1} & \text { for } i<c-1, & q_{c-1, c}=q^{-1} .
\end{array}
$$

One easily sees that $\mathscr{Y}=k$. A case-by-case study (according to the values of $i$ for which $d_{i}=n_{i}-1$ ) shows that the subspace $\mathrm{HH}_{\{1, \ldots, c-2\}}^{*}$ has a finite-dimensional complement in $\mathrm{HH}^{*}(\Lambda)$. It is nonempty if and only if $n_{c-1}=n_{c}$, and in that case

$$
\gamma\left(\mathrm{HH}^{*}(\Lambda)\right)=\gamma\left(\mathrm{HH}_{\{1, \ldots, c-2\}}^{*}\right)=\operatorname{pos} . \mathrm{rk} \operatorname{Ker} \underbrace{Q_{\{1, \ldots, c-2\} \times\{1, \ldots, c-2\}}}_{=0}=c-2 .
$$

Example 6.5. Let $q \in k^{\times}$not be a root of unity, let $c \in \mathbb{N}_{\geq 3}$, and (for simplicity) let char $k \neq 2$. Let $\Lambda$ be a quantum complete intersection of codimension $c$ with

$$
\begin{array}{llll}
q_{i j}=1 & \text { for } i, j<c-1, & q_{i, c-1}=q & \text { for } i<c-1, \\
q_{i c}=q^{-1} & \text { for } i<c-1, & q_{c-1, c}=q . &
\end{array}
$$

Then $\quad \mathscr{S}=\left\langle s_{p}\right| p_{i}$ even and $\left(Q\left(\frac{p_{j} n_{j}}{2}\right)_{j}\right)_{i}=1$ for all $\left.i\right\rangle$

$$
\left.=\left\langle s_{p}\right| p_{i} \text { even and } \sum_{j=0}^{c-2} p_{j} n_{j}=p_{c-1} n_{c-1}=p_{c} n_{c} \text { for all } i\right\rangle \text {. }
$$

In particular,

$$
\text { Krull.dim } \mathscr{Y}=c-2,
$$

and hence also $\gamma\left(\mathrm{HH}^{*}(\Lambda)\right)=c-2$, by Corollary 4.6 .

Similarly to Example 6.4 one sees that $\mathrm{HH}_{\{1, \ldots, c-2\}}^{*}+\mathrm{HH}_{\{1, \ldots, c\}}^{*}$ form a subspace of $\mathrm{HH}^{*}(\Lambda)$ which has a finite-dimensional complement. Since by Corollary 5.8 $\mathrm{HH}_{\{1, \ldots, c\}}^{*}$ is always finitely generated over $\mathscr{S}$, we only have to look at $\mathrm{HH}_{\{1, \ldots, c-2\}}^{*}$. As in Example 6.4, one sees that $\mathrm{HH}_{\{1, \ldots, c-2\}}^{*} \neq 0$ if and only if $n_{c-1}=n_{c}$. Since

pos.rk Ker $Q_{\{1, \ldots, c-2\} \times\{1, \ldots, c-2\}}=c-2 \neq 0=$ pos.rk $\operatorname{Ker} Q_{\{1, \ldots, c\} \times\{1, \ldots, c-2\}}$ it follows that $\mathrm{HH}^{*}(\Lambda)$ is finitely generated over $\mathscr{Y}$ if and only if $n_{c-1} \neq n_{c}$.

\section{Hochschild homology}

To calculate the Hochschild homology, we proceed as for the Hochschild cohomology. That is, we calculate for any $\boldsymbol{d} \in \mathbb{Z}^{c}$ the degree- $\boldsymbol{d}$ part of the Hochschild homology. The actual calculations are very similar to the corresponding ones in Section 3, and will therefore be omitted here.

Observation 7.1. The degree-d part $\left(\Lambda^{\mathrm{en}}\langle\boldsymbol{s}\rangle \otimes_{\Lambda^{\mathrm{en}}} \Lambda\right)_{\boldsymbol{d}}$ is nonzero if and only if $\boldsymbol{s} \leq \boldsymbol{d} \leq \boldsymbol{s}+\boldsymbol{n}-\mathbf{1}$. In that case it is one-dimensional. 
As in the case of the cohomology, it follows that the $c$-tuple complex

$$
\left(\mathbb{P}_{1} \otimes \cdots \otimes \mathbb{P}_{c} \otimes_{\Lambda^{\text {en }}} \Lambda\right)_{d}
$$

is concentrated in a cube (with sides of length 0 or 1), where there is a onedimensional space in each corner of the cube.

Next we need to understand what happens to a map $f: \Lambda^{\text {en }}\langle\boldsymbol{s}\rangle \rightarrow \Lambda^{\text {en }}\left\langle\boldsymbol{s}^{\prime}\right\rangle$ when it is tensored over $\Lambda^{\text {en }}$ with $\Lambda$.

Lemma 7.2. Let $f: \Lambda^{\mathrm{en}}\langle\boldsymbol{s}\rangle \rightarrow \Lambda^{\mathrm{en}}\left\langle\boldsymbol{s}^{\prime}\right\rangle$. Then $f \otimes_{\Lambda^{\mathrm{en}}} \Lambda: \Lambda\langle\boldsymbol{s}\rangle \rightarrow \Lambda\left\langle\boldsymbol{s}^{\prime}\right\rangle$ is given by

$$
\left(f \otimes_{\Lambda^{\text {en }}} \Lambda\right)\left(\boldsymbol{x}^{a}\right)=\sum_{i} f_{2}^{i} \boldsymbol{x}^{a} f_{1}^{i},
$$

where $f(1 \otimes 1)=\sum_{i} f_{1}^{i} \otimes f_{2}^{i}$.

Now we are ready to calculate what tensoring over $\Lambda^{\text {en }}$ with $\Lambda$ does to the maps occurring in the $c$-tuple complex $\mathbb{P}_{1} \otimes \cdots \otimes \mathbb{P}_{c}$.

Lemma 7.3. Let $\boldsymbol{s}$ and $\boldsymbol{d}$ be such that $\boldsymbol{s} \leq \boldsymbol{d} \leq \boldsymbol{s}+\boldsymbol{n}-\mathbf{1}$ and let $i \in\{1, \ldots, c\}$.

(1) Assume that $d_{i}>s_{i}$. The map $\left(\Lambda\left\langle s+e_{i}\right\rangle\right)_{d} \rightarrow(\Lambda\langle s\rangle)_{d}$ obtained by tensoring with $\Lambda$ the map over $\Lambda^{\text {en }}$ given by

$\Lambda^{\mathrm{en}}\left\langle\boldsymbol{s}+e_{i}\right\rangle \rightarrow \Lambda^{\mathrm{en}}\langle\boldsymbol{s}\rangle \quad$ mapping $\quad 1 \otimes 1 \mapsto \frac{1}{\boldsymbol{q}^{\left\langle e_{i} \mid \boldsymbol{s}\right\rangle}} x_{i} \otimes 1-\frac{1}{\boldsymbol{q}^{\left\langle\boldsymbol{s} \mid e_{i}\right\rangle}} 1 \otimes x_{i}$

and then taking the part of degree $\boldsymbol{d}$ vanishes if and only if $(Q d)_{i}=1$.

(2) Assume that $d_{i}=s_{i}+n_{i}-1$. The map $\left(\Lambda\left\langle s+\left(n_{i}-1\right) e_{i}\right\rangle\right)_{d} \rightarrow(\Lambda\langle s\rangle)_{d}$ obtained by tensoring with $\Lambda$ the map over $\Lambda^{\text {en }}$ given by

$$
\Lambda^{\mathrm{en}}\left\langle s+e_{i}\right\rangle \rightarrow \Lambda^{\mathrm{en}}\langle\boldsymbol{s}\rangle
$$

mapping

$$
1 \otimes 1 \mapsto \sum_{j=0}^{n_{i}-1}\left(\frac{1}{\boldsymbol{q}^{\left\langle e_{i} \mid \boldsymbol{s}\right\rangle}}\right)^{j}\left(\frac{1}{\boldsymbol{q}^{\left\langle\boldsymbol{s} \mid e_{i}\right\rangle}}\right)^{n_{i}-1-j} x_{i}^{j} \otimes x_{i}^{n_{i}-1-j}
$$

and then taking the part of degree $\boldsymbol{d}$ vanishes if and only if $(Q d)_{i} \in \mathscr{R}_{i}$.

As for the cohomology, it follows that if the map on one edge of the cube $\left(\mathbb{P}_{1} \otimes\right.$ $\left.\cdots \otimes \mathbb{P}_{c} \otimes_{\Lambda^{\text {en }}} \Lambda\right)_{d}$ vanishes then all parallel maps also vanish.

Theorem 7.4. Let $\Lambda=\Lambda_{q}^{\boldsymbol{n}}$ be a quantum complete intersection, and let $\boldsymbol{d} \in \mathbb{N}^{c}$. Divide the set $\{1 \ldots c\}$ into the three parts

$$
I_{0}=\left\{d_{i}=0\right\}, \quad I_{1}=\left\{n_{i} \mid d_{i}\right\} \backslash I_{0}, \quad I_{2}=\left\{n_{i} \nmid d_{i}\right\} .
$$

Then $\mathrm{HH}_{*, \boldsymbol{d}}(\Lambda) \neq 0$ if and only if

- $(Q d)_{i} \in \mathscr{R}_{i}$ for any $i \in I_{1}$, and

- $(Q d)_{i}=1$ for any $i \in I_{2}$. 
In this situation $\mathrm{HH}_{*, d}(\Lambda)$ has a k-vector space basis

$$
\left\{T_{\boldsymbol{d}}^{p} \mid \boldsymbol{p} \geq \mathbf{0} \text { and } \mathfrak{p}(\boldsymbol{d}+\mathbf{1})-\mathbf{2} \leq \boldsymbol{p} \leq \mathfrak{p}(\boldsymbol{d}+\mathbf{1})-\mathbf{1}\right\} .
$$

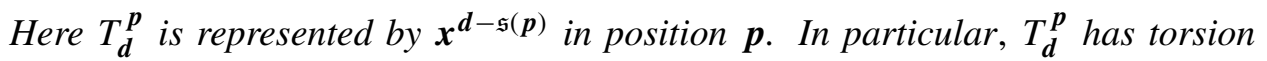
degree $\sum_{i=1}^{c} p_{i}$.

\section{The rate of growth of the Hochschild homology}

To study the rate of growth of the Hochschild homology, we decompose it similarly to our decomposition of the Hochschild cohomology in Construction 4.2.

Construction 8.1. For $G \subseteq\{1, \ldots, c\}$ we denote by $\mathrm{HH}_{*}^{G}$ the $k$-span of $T_{\boldsymbol{d}}^{p}$ with

$$
G=\left\{i \in\{1, \ldots, c\} \mid d_{i}>0 \text { or }(Q d)_{i} \in \mathscr{R}_{i}\right\} .
$$

This yields a decomposition $\mathrm{HH}_{*}(\Lambda)=\bigoplus_{G \subseteq\{1, \ldots, c\}} \mathrm{HH}_{*}^{G}$, and hence

$$
\gamma\left(\mathrm{HH}_{*}(\Lambda)\right)=\max _{G \subseteq\{1, \ldots, c\}} \gamma\left(\mathrm{HH}_{*}^{G}\right) .
$$

As in the proof of Theorem 4.5, one obtains:

Theorem 8.2. the rate of growth of the Hochschild homology of a finite-dimensional quantum complete intersection, is the maximum of pos.rk $\operatorname{Ker} Q_{G \times G}$ over

$$
G=\left\{i \in\{1, \ldots, c\} \mid d_{i}>0 \text { or }(Q d)_{i} \in \mathscr{R}_{i}\right\},
$$

where d ranges over element of $\mathbb{N}^{c}$ such that $(Q d)_{i} \in \mathscr{R}_{i}$ for all $i$ with $n_{i} \mid d_{i}$ and $d_{i}>0$, and $(Q d)_{i}=1$ for all $i$ with $n_{i} \nmid d_{i}$.

We conclude this paper by showing that the Hochschild homology of $\Lambda$ is closely related to the Hochschild homologies of certain subalgebras.

For $I \subset\{1, \ldots, c\}$, denote by $\Lambda_{I}$ the subalgebra of $\Lambda$ generated by $x_{i}$ with $i \in I$. Then $\Lambda_{I}$ is a split quotient of $\Lambda$ (that is, we have algebra homomorphisms $\Lambda_{I} \rightarrow \Lambda \rightarrow \Lambda_{I}$ whose composition is the identity on $\left.\Lambda_{I}\right)$. It follows from the functoriality of Hochschild homology that $\mathrm{HH}_{*}\left(\Lambda_{I}\right)$ can be embedded into $\mathrm{HH}_{*}(\Lambda)$.

The following theorem shows that the Hochschild homologies of these subalgebras determine the Hochschild homology of $\Lambda$ to a large extent.

Theorem 8.3. Let $M$ be the maximum of the rates of growth of $\mathrm{HH}_{*}\left(\Lambda_{\overline{\{i\}}}\right)$, where $i \in\{1, \ldots, c\}$ and $\overline{\{i\}}=\{1, \ldots, c\} \backslash\{i\}$. Then the rate of growth of $\mathrm{HH}_{*}(\Lambda)$ is $M$ if $\mathrm{HH}_{*}^{\{1, \ldots, c\}}=0$, and $\max \{M$, pos.rk $\operatorname{Ker} Q\}$ if $\mathrm{HH}_{*}^{\{1, \ldots, c\}} \neq 0$.

Proof. We will need to look at the sets $\mathrm{HH}_{*}^{G}$ as well as their analogs for $\mathrm{HH}_{*}\left(\Lambda_{\overline{\{i\}}}\right)$. To avoid confusion we write $\operatorname{HH}_{*}^{G}(\Lambda)$ and $\operatorname{HH}_{*}^{G}\left(\Lambda_{\overline{\{i\}}}\right)$, respectively, for these vector spaces. 
Let $i_{0} \in\{1, \ldots, c\}$ and $G \subseteq \overline{\left\{i_{0}\right\}}$. It follows from the explicit description of bases in Theorem 7.4 and Construction 8.1 that $\operatorname{HH}_{*}^{G}(\Lambda)$ can be identified with a subspace of $\mathrm{HH}_{*}^{G}\left(\Lambda_{\left.\overline{\left\{i_{0}\right.}\right\}}\right)$, and that the set of $T_{\boldsymbol{d}}^{p}$ such that

$$
G=\left\{i \in \overline{\left\{i_{0}\right\}} \mid d_{i}>0 \text { or }(Q d)_{i} \in \mathscr{R}_{i}\right\}
$$

and

$$
G \neq\left\{i \in\{1, \ldots, c\} \mid d_{i}>0 \text { or }(Q d)_{i} \in \mathscr{R}_{i}\right\}
$$

is a basis of the quotient space. This clearly means that

$$
\left\{i \in\{1, \ldots, c\} \mid d_{i}>0 \text { or }(Q d)_{i} \in \mathscr{R}_{i}\right\}=G \cup\left\{i_{0}\right\},
$$

so the quotient embeds naturally into $\mathrm{HH}_{*}^{G \cup\left\{i_{0}\right\}}$.

It follows that

$$
\gamma\left(\mathrm{HH}_{*}^{G}(\Lambda)\right) \leq \gamma\left(\mathrm{HH}_{*}^{G}\left(\Lambda_{\overline{\left\{i_{0}\right\}}}\right)\right) \leq \max \left\{\gamma\left(\mathrm{HH}_{*}^{G}(\Lambda)\right), \gamma\left(\mathrm{HH}_{*}^{G \cup\left\{i_{0}\right\}}(\Lambda)\right)\right\} .
$$

Taking the maximum over all $G$ and $i_{0}$ proves the theorem.

\section{References}

[Avramov et al. 1997] L. L. Avramov, V. N. Gasharov, and I. V. Peeva, "Complete intersection dimension”, Inst. Hautes Études Sci. Publ. Math. 86 (1997), 67-114. MR 99c:13033 Zbl 0918.13008

[Bergh and Erdmann 2008] P. A. Bergh and K. Erdmann, "Homology and cohomology of quantum complete intersections", Algebra Number Theory 2:5 (2008), 501-522. MR 2009h:16010 Zbl 05529335

[Bergh and Oppermann 2008a] P. A. Bergh and S. Oppermann, "Cohomology of twisted tensor products”, J. Algebra 320:8 (2008), 3327-3338. MR 2009f:16012 Zbl 1185.16012

[Bergh and Oppermann 2008b] P. A. Bergh and S. Oppermann, "The representation dimension of quantum complete intersections", J. Algebra 320:1 (2008), 354-368. MR 2009c:16020 Zbl 1163. 16008

[Buchweitz et al. 2005] R.-O. Buchweitz, E. L. Green, D. Madsen, and Ø. Solberg, "Finite Hochschild cohomology without finite global dimension”, Math. Res. Lett. 12:5-6 (2005), 805-816. MR 2006k:16019 Zbl 1138.16003

[Manin 1987] Y. I. Manin, "Some remarks on Koszul algebras and quantum groups", Ann. Inst. Fourier (Grenoble) 37:4 (1987), 191-205. MR 89e:16022 Zbl 0625.58040

[Yoneda 1958] N. Yoneda, "Note on products in Ext", Proc. Amer. Math. Soc. 9 (1958), 873-875. MR 31 \#233 Zbl 0101.27204

Communicated by Dave Benson

Received 2008-11-26 Revised 2010-08-27 Accepted 2010-10-01

steffen.oppermann@math.ntnu.no Institutt for matematiske fag, Norwegian University of Science and Technology, 7491 Trondheim, Norway http://www.math.ntnu.no/ opperman/ 


\section{Algebra \& Number Theory}

www.jant.org

\section{EDITORS}

\section{MANAGING EDITOR}

Bjorn Poonen

Massachusetts Institute of Technology

Cambridge, USA

\author{
EDITORIAL BOARD CHAIR \\ David Eisenbud \\ University of California \\ Berkeley, USA
}

\section{BOARD OF EDITORS}

\section{Georgia Benkart}

Dave Benson

Richard E. Borcherds

John H. Coates

J-L. Colliot-Thélène

Brian D. Conrad

Hélène Esnault

Hubert Flenner

Edward Frenkel

Andrew Granville

Joseph Gubeladze

Ehud Hrushovski

Craig Huneke

Mikhail Kapranov

Yujiro Kawamata

János Kollár

Hendrik W. Lenstra

Yuri Manin

Barry Mazur
University of Wisconsin, Madison, USA

University of Aberdeen, Scotland

University of California, Berkeley, USA

University of Cambridge, UK

CNRS, Université Paris-Sud, France

University of Michigan, USA

Universität Duisburg-Essen, Germany

Ruhr-Universität, Germany

University of California, Berkeley, USA

Université de Montréal, Canada

San Francisco State University, USA

Hebrew University, Israel

University of Kansas, USA

Yale University, USA

University of Tokyo, Japan

Princeton University, USA

Universiteit Leiden, The Netherlands

Northwestern University, USA

Harvard University, USA
Susan Montgomery

Shigefumi Mori

Andrei Okounkov

Raman Parimala

Victor Reiner

Karl Rubin

Peter Sarnak

Michael Singer

Ronald Solomon

Vasudevan Srinivas

J. Toby Stafford

Bernd Sturmfels

Richard Taylor

Ravi Vakil

Michel van den Bergh

Marie-France Vignéras

Kei-Ichi Watanabe

Andrei Zelevinsky

Efim Zelmanov
University of Southern California, USA

RIMS, Kyoto University, Japan

Princeton University, USA

Emory University, USA

University of Minnesota, USA

University of California, Irvine, USA

Princeton University, USA

North Carolina State University, USA

Ohio State University, USA

Tata Inst. of Fund. Research, India

University of Michigan, USA

University of California, Berkeley, USA

Harvard University, USA

Stanford University, USA

Hasselt University, Belgium

Université Paris VII, France

Nihon University, Japan

Northeastern University, USA

University of California, San Diego, USA

\section{PRODUCTION}

ant@mathscipub.org

Silvio Levy, Scientific Editor

Andrew Levy, Production Editor

See inside back cover or www.jant.org for submission instructions.

The subscription price for 2010 is US \$140/year for the electronic version, and \$200/year (+\$30 shipping outside the US) for print and electronic. Subscriptions, requests for back issues from the last three years and changes of subscribers address should be sent to Mathematical Sciences Publishers, Department of Mathematics, University of California, Berkeley, CA 94720-3840, USA.

Algebra \& Number Theory (ISSN 1937-0652) at Mathematical Sciences Publishers, Department of Mathematics, University of California, Berkeley, CA 94720-3840 is published continuously online. Periodical rate postage paid at Berkeley, CA 94704, and additional mailing offices.

ANT peer review and production are managed by EditFLOW ${ }^{\mathrm{TM}}$ from Mathematical Sciences Publishers.

\section{PUBLISHED BY}

mathematical sciences publishers

http://www.mathscipub.org

A NON-PROFIT CORPORATION

Typeset in LATEX

Copyright $\odot 2010$ by Mathematical Sciences Publishers 


\section{Algebra \& Number Theory}

Volume $4 \quad$ No. $7 \quad 2010$

Hochschild cohomology and homology of quantum complete intersections

STEFFEN OPPERMANN

Meromorphic continuation for the zeta function of a Dwork hypersurface

THOMAS BARNET-LAMB

Equations for Chow and Hilbert quotients

ANGEla Gibney and Diane MaClagan

Haglund-Haiman-Loehr type formulas for Hall-Littlewood polynomials of type $B$ and C

\section{CRISTIAN LENART}

On exponentials of exponential generating series

ROLAND BACHER

On families of $\varphi, \Gamma$-modules

KIRAN KEDLAYA and RUOCHUAN LIU 